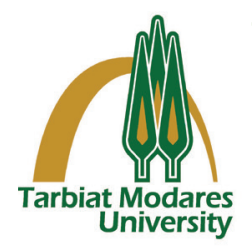

\title{
Frequency and Antibiotic Resistance of Hybrid Entroaggregative/Uropathogenic Escherichia coli Isolated from Patients Hospitalized in Isfahan, Iran
}

\section{A R T I C L E I N F O}

Article Type

Original Research

Authors

Tahere Alipour, $M S c^{1}$

Sorour Farzi, $M S c^{2}$

Amir Emamie, $M S c^{3}$

Atieh Darbandi, $\mathrm{PhD}^{* 4}$

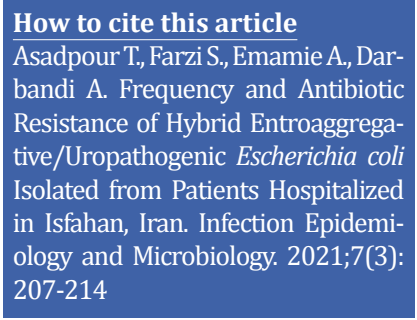

${ }^{1}$ Department of Bacteriology and Virology, School of Medicine, Isfahan University of Medical Sciences, Isfahan, Iran.

2Department of Microbiology, Faculty of Medicine, Shahed University, Tehran, Iran. ${ }^{3}$ Department of Pathobiology, School of Public Health, Tehran University of Medical Sciences, Tehran, Iran

${ }^{4}$ Department of Microbiology, School of Medicine, Iran University of Medical Sciences, Tehran, Iran.

\footnotetext{
* Correspondence

Address: Atieh Darbandi, Department of Microbiology, School of Medicine and Microbial Biotechnology Research Centre, Iran University of Medical Sciences, Shahid Hemmat Highway, Tehran, Iran. Darbandi.a@iums.ac.ir
}

\begin{abstract}
A B S T R A C T
Backgrounds: This study aimed to analyse hybrid Entroaggregative/Uropathogenic Escherichia coli (EAEC/UPEC) isolates. To do so, the antibiotic resistance pattern and virulence genes were investigated in E. coli strains isolated from clinical specimens of patients hospitalized in Isfahan, Iran.

Materials \& Methods: Disc diffusion method was used to determine the antibiotic susceptibility pattern of EAEC/UPEC isolates. Also, virulence determinants of these isolates were determinated by singleplex and multiplex PCR.

Findings: Overall, a total of 148 E. coli isolates were collected, of which 12 (8.1\%) isolates were hybrid EAEC/UPEC strains, then antibiotic susceptibility examination was operated on these strains. The higest antibiotic resistance rate was related to ofloxacin (42\%), followed by trimethoprim-sulfamethoxazole (41\%), ceftriaxone and cefepime $(33 \%)$, and cefoxitin (17\%). All the isolates showed susceptibility to fosfomycin.

Conclusion: According to the current study, since resistance to fluoroquinolones has increased in hybrid strains, monitoring the drug susceptibility of hybrid strains seems critical in Iran. Fosfomycin is considered to be the drug of choise for infections caused by multidrug-resistant (MDR) Gram-positive and Gram-negative bacteria. Fortunately, $100 \%$ of the strains were sensitive to fosfomycin.
\end{abstract}

Keywords: Enteroaggregative Escherichia coli, Uropathogenic Escherichia coli, Hybrid strains.

\section{CITATION LINKS}

[1] Soltani S, Emamie AD, Dastranj M, Farahani A, Davoodabadi ... [2] Santos ACdM, Santos F, Silva RM, Gomes TAT. Diversity of ... [3] Miri ST, Dashti A, Mostaan S, Kazemi F, Bouzari S. Identification of ... [4] Jensen BH, Olsen KE, Struve C, ... [5] Momtaz H, Karimian A, Madani M, Dehkordi FS, Ranjbar R, Sarshar M, et al. Uropathogenic ... [6] Johnson JR. Virulence factors in... [7] Soto S, Guiral E, ... [8] Bauer RJ, Zhang L, Foxman B, Siitonen A, Jantunen ME, ... [9] Jahandeh N, Ranjbar R, Behzadi P, Behzadi E. Uropathogenic Escherichia coli ... [10] Farshad S, Ranjbar R, Japoni A, Hosseini M, Anvarinejad ... [11] Tajbakhsh E, Khamesipour F, Ranjbar R, ... [12] Davies J, Davies D. Origins and evolution of Blair JMA, Webber MA, Baylay AJ, Ogbolu DO, Piddock LJ. Molecular ... [13] Blair JMA, Webber MA, Baylay AJ .... [14] Boll EJ, Struve C, Boisen N, Olesen B, Stahlhut SG, Krogfelt KA. Role of .... [15] Mohebi S, Hossieni Nave H, Norouzi A, ... [16] Toval F, Köhler C-D, Vogel U, Wagenlehner F, Mellmann A, Fruth A, et al. ... [17] Santos ACdM, Santos F, Silva RM, Gomes TAT. Diversity of hybrid-and hetero-pathogenic ... [18] Darbandi A, Owila P, Bouzari S, Saderi H. Diarrheagenic Escherichia ... [19] Shahbazi S, Karam MRA, Habibi M, Talebi A, Bouzari S. Distribution of ... [20] Nave HH, Mansouri S, Moghadam MT, Moradi M. Virulence ... [21] Kot B. Antibiotic resistance among uropathogenic ... [22] Alikhani MY, Hashemi ... [23] Bouzari S, Jafari A, Zarepoor M. Distribution of genes ... [24] Clinical and Laboratory ... [25] Kot B. Antibiotic ... [26] Najafi N, Alikhani A, Babamahmoudi F, Davoudi A, Ghasemiyan R, Aliyan S, et al. Increased ... [27] Farajnia S, Alikhani MY, Ghotaslou R, ... [28] Adib N, Ghanbarpour R, Solatzadeh H, Alizade H. Antibiotic ... [29] Alikhani A, Babamahmoodi F, Alizadegan LF, Shojaeefar A, Babamahmoodi A. Minimal inhibitory ... [30] Park YS, Adams-Haduch JM, Shutt KA, Yarabinec III DM, Johnson LE, Hingwe A, et al. Clinical ... [31] Hawser S, Bouchillon S, Lascols C, Hackel M, ... [32] Bantar C, Famiglietti A, Goldberg M, Group NS. Three-year surveillance ... [33] Shakya P, Barrett P, Diwan V, Marothi Y, Shah H, Chhari N, et al. Antibiotic resistance ... [34] Samal SK, Khuntia HK, Nanda PK, Satapathy CS, Nayak SR, Sarangi AK, et al. Incidence ... [35] Vaishnavi C, Kaur S. The ... [36] Falagas ME, Kastoris AC, Karageorgopoulos DE, Rafailidis ... [37] Neuner EA, Sekeres J, Hall GS, van Duin D. Experience with ... [38] Falagas ME, Kastoris AC, Kapaskelis AM, Karageorgopoulos DE. .... [39] Bahramian A, Eslami G, Hashemi A, Tabibi A, Heidary M. Emergence of ... [40] Malekpour Kolbadinezhad S, Fozouni L. Molecular monitoring of ... [41] Cottell JL, Webber MA. Experiences in fosfomycin susceptibility ... [42] Bader MS, Loeb M, Brooks ...

\section{Article History}

Received: May 25,2021

Accepted: July 05,2021

Published: July 20,2021

Copyright(C) 2021, TMU Press. This open-access article is published under the terms of the Creative Commons Attribution-NonCommercial 4.0 International License which permits Share (copy and redistribute the material in any medium or format) and Adapt (remix, transform, and build upon the material) under the Attribution-NonCommercial terms. 


\section{Introduction}

Escherichia coli is a commensal bacterium that colonizes humans shortly after birth. It has also been reported to interact with its hosts ${ }^{[1]}$. However, they are circumscribed to the outside bed of the intestine, which allows them to sometimes cause enteric or extraintestinal pathological processes in humans. Some extremely altered $E$. coli strains have appeared during their development. Gaining extensive virulence factors, E. coli is expected to change, colonize, and invade many body sites ${ }^{[2-3]}$

Enteroaggregative E. coli (EAEC) is amongst the foremost notable heterogeneous strains of $E$. coli and is a causative agent of persistent watery diarrhea in children and adults worldwide ${ }^{[4]}$.

Uropathogenic E. coli (UPEC) is a strain recovered from urinary tract infections (UTIs) and is the leading cause of bacterial infections amongst humans, accounting for 100 million UTL cases per year. These strains are a heterogeneous category of $E$. coli strains harboring a significant number of well-recognized virulence factors (VFs) expressed in various genotypes [5].

In EAEC, the transcriptional activator $\operatorname{agg} R$, involved in the launch of a minimum of 44 genes, regulates both plasmid and chromosomal AAF gene expression ${ }^{[6-7]}$. Some virulence factors of UPEC strains include aerobactin (aer), P fimbriae (pap), hemin receptor $(c h u A)$, yersiniabactin siderophore receptor $(f y u A)$, hemolysin (hly), type 1 fimbriae, afimbrial adhesin I ( $a f a I)$, cytotoxic necrotizing factor 1 (cnf1), S fimbriae ( $s f a$ ), adhesins, and fimbriae [8-10].

Recently, the frequency of antibiotic resistance in urinary tract pathogens has increased [11-12]. Mobile genetic elements (MGEs) including transposons, plasmids, and integrons are the most important factors for transferring resistance genes among bacteria. E. coli strains may be intrinsically resistant to antibiotics and may harbor genes responsible for resistance to antibiotics like aminoglycosides, fluoroquinolones, and $\beta$-lactams [13-14].

Interestingly, hybrid EAEC/UPEC pathotypes are considered as the causative agents of UTIs, afterward resulting in bacteremia and sepsis in patients [15-17]. Studies have shown concerns about the increased isolation of EAEC and UPEC pathotypes carrying virulence genes. Also, they have shown resistance of these isolates to several antimicrobials commonly used for treatment [18-19]. Some studies have investigated the genomic characterization of hybrid EAEC/ UPEC and virulence factors among different pathotypes isolated.

Objectives: This study aimed to investigate hybrid EAEC/UPEC strains isolated from clinical samples to identify their virulence genes and antibiotic susceptibility patterns in Isfahan, Iran.

\section{Materials \& Methods}

sample collection and identification: In this study, a total of 148 E. coli isolates were recovered from patients admitted to three hospitals (Imam Hossain, Shariati, and Al-Zahra hospitals) in Isfahan from July to November 2019. Samples were collected from urine, stool, biopsy, blood, and wounds from different hospital wards, such as pediatric, surgical, ICU, emergency, internal, and outpatient. Then the collected samples were immediately transported to the laboratory and stored at $4{ }^{\circ} \mathrm{C}$ for further analysis.

Microbial Identification: Collected samples were cultured on selective media such as MacConkey agar, Eosin methylene blue (EMB), and xylose lysine deoxycholate (XLD) (Merck KGaA, Darmstadt, Germany) at $37{ }^{\circ} \mathrm{C}$ for $24-48$ hours. After overnight incubation, all lactose-positive colonies were re-cultured, and then standard phenotypic 
and biochemical tests were performed and analyzed on triple sugar iron (TSI) agar (Merck KGaA, Darmstadt, Germany), SIM (Condalab, Madrid, Spain), MR-VP, urea broth, Simmon citrate agar, and Lauryl sulfate broth (LSB) (Biolife, Milan, Italy) to identify the isolated bacteria. Confirmed $E$. coli isolates were stored in brain heart infusion (BHI) broth (HiMedia Laboratories, Mumbai, India) comprising 20\% glycerol (Merck KGaA, Darmstadt, Germany) at $-20{ }^{\circ} \mathrm{C}$ for detecting EAEC and UPEC pathotypes ${ }^{[20]}$. Reference strain E. coli ATCC 25922 was used as quality control in each assay. The reference strain was supplied from the Bacterial Cell Bank of Pasteur Institute of Iran (CSBPI).

Examining hybrid EAEC/UPEC and virulence agents: In order for detecting hybrid EAEC/UPEC strains, PCR methods was carried out. A singleplex PCR was executed to amplify fimH, chu, fyuA, and papc genes, and multiplex PCR was operated to detect $a p p, a g g R$, and aatA genes. PCR amplification was performed in a PCR mixture containing 10 pmol of each primer, $12.5 \mu \mathrm{L}$ of Qiagen HotStar Taq polymerase Master Mix 1X, $3 \mu \mathrm{L}$ of extracted DNA, and sterile distilled water. PCR methods were performed as previously described ${ }^{[21-23]}$. To verify the presence and sequencing of VGs in hybrid EAEC/UPEC pathotypes, PCR amplicons were sent to Niagenenoor Company (Iran, Tehran). Online BLAST software was employed to examine the sequences in NCBI database (https://www.ncbi.nlm.nih.gov/BLAST/). Antimicrobial susceptibility experiment: The disc diffusion method was employed for in vitro antimicrobial susceptibility testing of $E$. coli isolates as suggested by the Clinical and Laboratory Standards Institute (CLSI) ${ }^{[24]}$. The following antibiotic disks on Mueller Hinton agar (MAST Categories Ltd., Merseyside, U.K.) were used: cefoxitin (30 $\mu \mathrm{g})$, ofloxacin $(5 \mu \mathrm{g})$, ceftriaxone $(30 \mu \mathrm{g})$, fosfomycin $(200 \mu \mathrm{g})$, cefepime $(30 \mu \mathrm{g})$, and trimethoprim-sulfamethoxazole $(25 \mu \mathrm{g})$. they were then diluted to 0.5 McFarland turbidity standards. A sterile cotton swab was inserted into the standardized inoculum and inoculated evenly on Mueller-Hinton agar (Merck, Germany). The plate was then incubated at $37{ }^{\circ} \mathrm{C}$ for $24-18$ hours. The zones of growth inhibition were investigated. Multidrug-resistant (MDR) and extensively drug-resistant (XDR) strains were isolated according to criteria characterized by the standardized international terminology explained by the Centre for Disease Prevention and Control (CDC) and therefore the European Centre for Disease Prevention and Control (ECDC) ${ }^{[25]}$. The reference strain E. coli ATCC 25922 was used as a control in all steps.

Statistical analysis methods: Data were analysed in SPSS software, Version 18.0 (IBM Corp., Armonk, USA). Descriptive statistics were carried out on the data collected. Categorical determinants were analysed using the Chi-square $(\chi 2)$ or Fisher's exact test. The $p$ values $<.05$ were considered statistically significant.

\section{Findings}

In the present study, a total of 148 E. coli strains were isolated and verified using confirmatory tests. Of which 62 (41\%) strains were collected from patients with extra-intestinal infections, including bacteremia ( $4 ; 6.4 \%)$, UTI $(57 ; 91 \%)$, and wound infection $(1 ; 1.6 \%)$. Besides, 86 (58\%) isolates were collected from colon biopsies (8; 9.3\%) and stool specimens (78; $90.7 \%$ ) of patients with enteric infections (Figure 1).

To detect hybrid EAEC/UPEC strains, singleplex PCR was carried out to amplify the fimH, chu, fyuA, and papC genes, and multiplex PCR was done to detect the $a p p$ $\operatorname{aggR,}$ and aatA genes.

There was a significant relationship between 


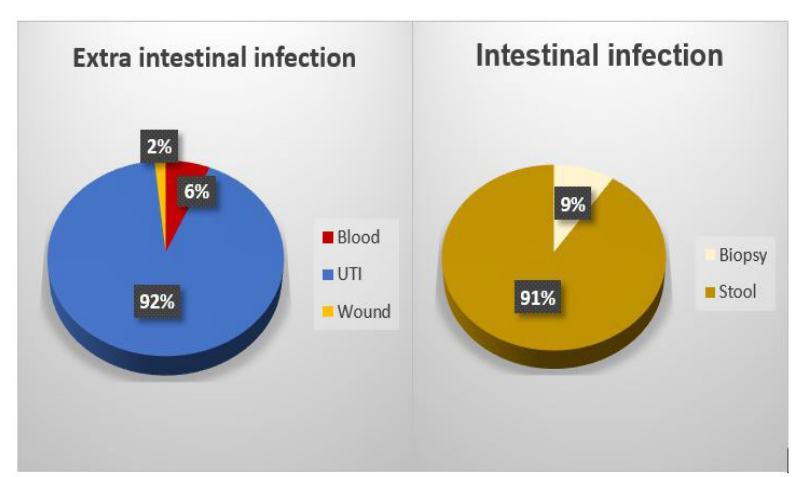

Figure 1) Percentage of E. coli strains isolated among different samples

VGs and hybrid EAEC/UPEC pathotypes $(p<.05)$. There was also a remarkable relation between the presence of $p a p C$ and fyuA genes and $E$. coli isolates $(p<.05)$; however, no significant relationship was found for the presence of $c h u A$, fimH, aatA, aggR, and app genes $(p>05)$. BLAST sequencing analyses confirmed the presence of aatA, app, aggR, $p a p C$, fim $H, c h u A$, and fyu $A$ genes. In addition to the $\operatorname{aggR}$, aap, and aatA genes, isolates carryed the UPEC-specific genes, including chuA, fyuA, fimH, and papC, highlighting their hybrid nature as hybrid EAEC/UPEC isolates.

In this study, 148 E. coli were collected, among them 12 (8.1\%) isolates were hybrid strains. The hybrid EAEC/UPEC strains were tested against the antibiotic disks mentioned above on Mueller Hinton agar (Merck KGaA, Darmstadt, Germany). Most hybrid strains were resistant to ofloxacin (42\%) and trimethoprim-sulfamethoxazole (41\%), many were resistant to ceftriaxone and cefepime (33\%), and lower than $20 \%$ were resistant to cefoxitin. More details are represented in Figure 2.

In addition to the antibiotic resistance pattern, XDR and MDR strains were identified; $8.3 \%$ (1 of 12 ) and $16.6 \%$ (2 of 12 ) of the isolates were MDR and XDR.

\section{Discussion}

This study analyzed the prevalence of virulence determinants and antibiotic

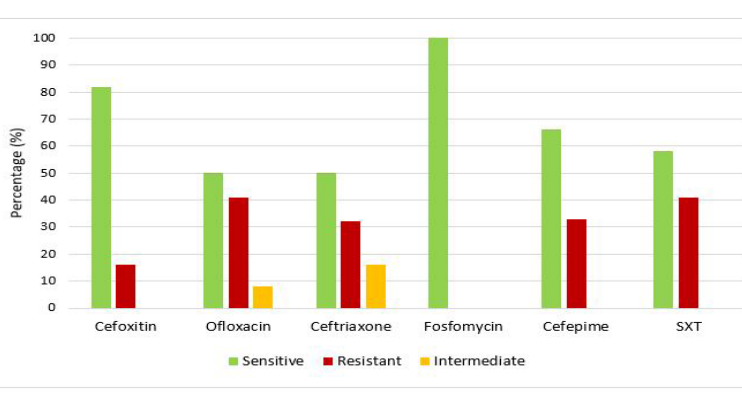

Figure 2) Phenotypic antibiogram profile of the hybrid EAEC/UPEC strains isolated from clinical specimens. SXT: Trimethoprim/Sulfamethoxazole

susceptibility pattern of the hybrid EAEC/ EUEC isolates obtained from patients referreing to three major hospitals in Isfahan. Many studies have evaluated the prevalence of cefepime-resistant $E$. coli strains in Iran. The lowest (25\%; 95\%CI: 21.67-28.55) and highest (61.95\%; 95\%CI: 56.62-67.07) prevalence of hybrid EAEC/UPEC strains has been observed in Mazandaran and East Azerbaijan, respectively [26-27].

In recent years, the increasing rates of antibiotic resistance imply a severe health problem with limited empirical treatment options, especially for UTIs. Therefore, the challenge of antibiotic resistance requires an imperative response to reduce the overuse of antibiotics.

The resistance rate of these hybrid strains to antibiotics, such as ampicillin, tetracycline, amikacin, and nitrofurantoin, has been reported to be high; therefore, these antibiotics should not be prescribed as firstline therapeutic drugs for Enterobacteriaceae [28-29]. In this study, antibiotic susceptibility testing was performed against cefoxitin, ofloxacin, ceftriaxone, fosfomycin, cefepime, and trimethoprim-sulfamethoxazole. Interestingly, despite the high antimicrobial resistance amongst the hybrid EAEC/EUEC strains, these organisms were susceptible to fosfomycin (100\%), cefoxitin (83\%), and Trimethoprim-sulfamethoxazole (58\%), which are the drugs of choice in many countries ${ }^{[30]}$. 
The prevalence of cefepime-resistant E. coli isolates in Iran has been shown to vary from 15.3 to $100 \%{ }^{[30-32]}$. Also, the prevalence of cefepime-resistant $E$. coli strains differs in various countries globally. Overall, the estimated frequency of cefepime resistance amongst E. coli isolates is as follows: $10.3 \%$ in the U.S., 8.8\% in Europe, $6 \%$ in Argentina, and $13 \%$ in India ${ }^{[33-34]}$, which is consistent with this study result.

In this study, $50 \%$ of the isolates were susceptible to fluoroquinolones, such as ofloxacin, commonly used as firstline antibiotics to treat diarrhea caused by hybrid EAEC/EUEC strains. Indeed, several studies have examined the spread of fluoroquinolone-resistant intestinal pathogens ${ }^{[35-36]}$. Thus, evaluation of antibiotic susceptibility of EAEC/EUEC hybrid strains seems to be a significant matter in Iran. Also, fosfomycin has been successfully figured out as a drug of choice for infections caused by MDR Gram-negative and Gram-positive organisms [37-38]. A study conducted on clinical MDR Enterobacteriaceae isolates, including extended-spectrum $\beta$-lactamase (ESBL) producing $E$. coli isolates, showed that over $90 \%$ of isolates were susceptible to fosfomycin [38]. Similarly in the present research, $100 \%$ of the hybrid EAEC/UPEC isolates were susceptible to fosfomycin. The rate of resistance to this antibiotic in some studies conducted in Iran varies from $6.6 \%{ }^{[39]}$ to $15 \%{ }^{[40]}$. The rate of fosfomycin resistance is low worldwide. In England, resistance to fosfomycin has been reported to be $9.5 \%$

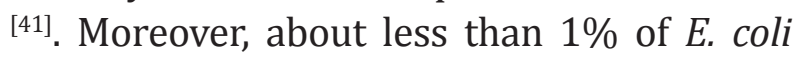
strains isolated in clinical laboratories in Canada were shown in a study to be resistant to fosfomycin ${ }^{[42]}$.

\section{Conclusion}

According to this study, drug resistance and multidrug resistance among E. coli strains causing UTIs have increased in recent years. This increase may be due to misuse and overuse of antibiotics without antibiotic susceptibility testing and without considering several factors such as age, sex, and health conditions in various geographical regions. In the present study, the hybrid EAEC/UPEC strains showed high sensitivity to cefoxitin (83\%) and fosfomycin (100\%). These two antibiotics still seem to be appropriate drugs for the treatment of patients with infections caused by hybrid EAEC/UPEC strains.

Acknowledgment: The authors kindly thank the Student Research Committee of Isfahan University of Medical Sciences for supporting this study.

Ethical Permission: This work was approved by Ethical committee of our University.

Conflict of interest: The authors announce that they have no competing interests.

Author's contribution: Conceptualization: TA and AD; Data curation and formal analysis: TA and SF; Investigation: TA, SF and AD; Methodology and project administration: AD; Supervision: AD; Validation: AD; Writing of original draft: AE and TA; Writing, reviewing and editing: $\mathrm{AD}$ and $\mathrm{AE}$.

Fundings: The researchers did not receive any specific grants from funding agencies in the public, commercial, or not-for-profit sectors.

Consent to participate: Consent form was obtained from all healthy people.

\section{Reference}

1. Soltani S, Emamie AD, Dastranj M, Farahani A, Davoodabadi A, Mohajeri P. Role of toxins of uropathogenic Escherichia coli in development of urinary tract infection. J Pharm Res Int. 2018;21(1):1-11.

2. Santos ACdM, Santos F, Silva RM, Gomes TAT. Diversity of hybrid-and hetero- 
pathogenic Escherichia coli and their potential implication in more severe diseases. Front Cell Infect Microbiol. 2020;10:339.

3. Miri ST, Dashti A, Mostaan S, Kazemi F, Bouzari S. Identification of different Escherichia coli pathotypes in north and north-west provinces of Iran. Iran J Microbiol. 2017;9(1):33-7.

4. Jensen BH, Olsen KE, Struve C, Krogfelt KA, Petersen AM. Epidemiology and clinical manifestation of enteroaggregative Escherichia coli. Clin Microbiol Rev. 2014;27(3):614-30.

5. Momtaz H, Karimian A, Madani M, Dehkordi FS, Ranjbar R, Sarshar M, et al. Uropathogenic Escherichia coli in Iran: Serogroup distributions, virulence factors, and antimicrobial resistance properties. Ann Clin Microbiol Antimicrob. 2013;12(1):1-2.Johnson JR. Virulence factors in Escherichia coli urinary tract infection. Clin Microbiol Rev. 1991;4(1):80-128.

6. Soto S, Guiral E, Bosch J, Vila J. Prevalence of the set-1B and ast $A$ genes encoding enterotoxins in uropathogenic Escherichia coli clinical isolates. Microb Pathog. 2009;47(6):305-7.

7. Bauer RJ, Zhang L, Foxman B, Siitonen A, Jantunen ME, Saxen H, et al. Molecular epidemiology of 3 putative virulence genes for Escherichia coli urinary tract infection-usp, iha, and iroNE. coli. J Infect Dis. 2002;185(10):1521-4.

8. Jahandeh N, Ranjbar R, Behzadi P, Behzadi E. Uropathogenic Escherichia coli virulence genes: Invaluable approaches for designing DNA microarray probes. Cent European J Urol. 2015;68(4):452-8.

9. Farshad S, Ranjbar R, Japoni A, Hosseini M, Anvarinejad M, Mohammadzadegan R. Microbial susceptibility, virulence factors, and plasmid profiles of uropathogenic Escherichia coli strains isolated from children in Jahrom, Iran. Arch Iran Med. 2012;15(5):312-6.

10. Tajbakhsh E, Khamesipour F, Ranjbar R, Ugwu IC. Prevalence of class 1 and 2 integrons in multi-drug resistant Escherichia coli isolated from aquaculture water in Chaharmahal Va Bakhtiari province, Iran. Ann Clin Microbiol Antimicrob. 2015;14(1):1-5. Davies J, Davies D. Origins and evolution of antibiotic resistance. Microbiol Mol Biol Rev. 2010;74(3):417-33.

11. Blair JMA, Webber MA, Baylay AJ, Ogbolu DO, Piddock LJ. Molecular mechanisms of antibiotic resistance. Nat Rev Microbiol. 2014;13(1):42-51.

12. Boll EJ, Struve C, Boisen N, Olesen B, Stahlhut SG, Krogfelt KA. Role of enteroaggregative Escherichia coli virulence factors in uropathogenesis. Infect Immun. 2013;81(4):1164-71.

13. Mohebi S, Hossieni Nave $H$, Norouzi A, Kandehkar Gharaman M, Taati Moghadam M. Detection of extended spectrum beta lactamases on class I integron in Escherichia coli isolated from clinical samples. J Mazandaran Univ Med Sci. 2016;26(138):66-76.

14. Toval F, Köhler C-D, Vogel U, Wagenlehner F, Mellmann A, Fruth A, et al. Characterization of Escherichia coli isolates from hospital inpatients or outpatients with urinary tract infection. J Clin Microbiol. 2014;52(2):407-18.

15. Santos ACdM, Santos F, Silva RM, Gomes TAT. Diversity of hybrid-and heteropathogenic Escherichia coli and their potential implication in more severe diseases. Front Cell Infect Microbiol. 2020;10:339.

16. Darbandi A, Owila P, Bouzari S, Saderi H. Diarrheagenic Escherichia coli pathotypes frequency in Khuzestan province of Iran. Iran j Microbiol. 2016;8(6):352-8. 
17. Shahbazi S, Karam MRA, Habibi M, Talebi A, Bouzari S. Distribution of extended-spectrum $\beta$-lactam, quinolone and carbapenem resistance genes, and genetic diversity among uropathogenic Escherichia coli isolates in Tehran, Iran. J Glob Antimicrob Resist. 2018;14:118-25.

18. Nave HH, Mansouri S, Moghadam MT, Moradi M. Virulence gene profile and multilocus variable-number tandemrepeat analysis (MLVA) of enteroinvasive Escherichia coli (EIEC) isolates from patients with diarrhea in Kerman, Iran. Jundishapur J Microbiol. 2016;9(6):1-6.

19. Kot B. Antibiotic resistance among uropathogenic Escherichia coli. Pol J Microbiol. 2019;68(4):403-15.

20. Alikhani MY, Hashemi SH, Aslani MM, Farajnia S. Prevalence and antibiotic resistance patterns of diarrheagenic Escherichia coli isolated from adolescents and adult in Hamedan,Western Iran. Iran J Microbial. 2013;5(1):42-7.

21. Bouzari S, Jafari A, Zarepoor M. Distribution of genes encoding toxins and antibiotic resistance patterns in diarrhoeagenic Escherichia coli isolates in Tehran. East Mediterr Health J. 2007;13(2):287-93.

22. Clinical and Laboratory Standards Institute. M100: Performance standards for antimicrobial susceptibility testing. 30th Ed. CLSI supplement. Wayne: Clinical and Laboratory Standards Institute; 2020.

23. Kot B. Antibiotic resistance among uropathogenic Escherichia coli. Pol J Microbiol. 2019;68(4):403-15.

24. Najafi N, Alikhani A, Babamahmoudi F, Davoudi A, Ghasemiyan R, Aliyan $\mathrm{S}$, et al. Increased cefepime MIC for enterobacteriacaeclinical isolates. Caspian J Intern Med. 2013;4(2):654-7.

25. Farajnia S, Alikhani MY, Ghotaslou R, Naghili B, Nakhlband A. Causative agents and antimicrobial susceptibilities of urinary tract infections in the northwest of Iran. Int J Infect Dis. 2009;13(2):1404.

26. Adib N, Ghanbarpour R, Solatzadeh H, Alizade H. Antibiotic resistance profile and virulence genes of uropathogenic Escherichia coli isolates in relation to phylogeny. Trop Biomed. 2014;31(1):1725.

27. Alikhani A, Babamahmoodi F, Alizadegan LF, Shojaeefar A, Babamahmoodi A. Minimal inhibitory concentration of microorganisms causing surgical site infection in referral hospitals in North of Iran, 2011-2012. Caspian J Intern Med. 2015;6(1):34-9.

28. Park YS, Adams-Haduch JM, Shutt KA, Yarabinec III DM, Johnson LE, Hingwe $A$, et al. Clinical and microbiologic characteristics of cephalosporinresistant Escherichia coli in three centers in the United States. Antimicrob Agents Chemother. 2012;56(4):1870-6.

29. Hawser S, Bouchillon S, Lascols C, Hackel M, Hoban DJ, Badal RE, etal.Susceptibility of European Escherichia coli clinical isolates from intra-abdominal infections, extended-spectrum $\quad \beta$-lactamase occurrence, resistance distribution, and molecular characterization of ertapenem-resistant isolates (SMART 2008-2009). Clin Microbiol Infect. 2012;18(3):253-9.

30. Bantar C, Famiglietti A, Goldberg M, Group NS. Three-year surveillance study of nosocomial bacterial resistance in Argentina. Int J Infect Dis. 2000;4(2):8590.

31. Shakya P, Barrett P, Diwan V, Marothi Y, Shah H, Chhari N, et al. Antibiotic resistance among Escherichia coli isolates from stool samples of children aged 3 to 14 years from Ujjain, India. BMC Infect Dis. 2013;13(1):1-6. 
32. Samal SK, Khuntia HK, Nanda PK, Satapathy CS, Nayak SR, Sarangi AK, et al. Incidence of bacterial enteropathogens among hospitalized diarrhea patients from Orissa, India. Jpn J Infect Dis. 2008;61(5):350-5.

33. Vaishnavi C, Kaur S. The epidemiological and resistogram patterns of enteropathogenic and enterotoxigenic Escherichia coli isolated from diarrhoeal stools in a north Indian hospital. Trop Gastroenterol. 2003;24(2):70-2.

34. FalagasME, KastorisAC, Karageorgopoulos DE, Rafailidis PI. Fosfomycin for the treatment of infections caused by multidrug-resistant non-fermenting gram-negative bacilli: A systematic review of microbiological, animal and clinical studies. Int J Antimicrob Agents. 2009;34(2):111-20.

35. Neuner EA, Sekeres J, Hall GS, van Duin D. Experience with fosfomycin for treatment of urinary tract infections due to multidrug-resistant organisms. Antimicrob Agents Chemother. 2012;56(11):5744-8.

36. Falagas ME, Kastoris AC, Kapaskelis AM, Karageorgopoulos DE. Fosfomycin for the treatment of multidrug-resistant, including extended-spectrum betalactamase producing, Enterobacteriaceae infections: A systematic review. Lancet Infect Dis. 2010;10(1):43-50.

37. Bahramian A, Eslami G, Hashemi A, Tabibi A, Heidary M. Emergence of fosfomycin resistance among isolates of Escherichia coli harboring extended-spectrum and AmpC $\beta$-lactamases. Acta Microbiol Immunol Hung. 2018;65(1):15-25.

38. Malekpour Kolbadinezhad S, Fozouni L. Molecular monitoring of fosfomycin resistance in Escherichia coli strains isolated from patients with urinary catheters in north-east of Iran. J Med Microbiol Infect Dis. 2018;6(4):112-7.

39. Cottell JL, Webber MA. Experiences in fosfomycin susceptibility testing and resistance mechanism determination in Escherichia coli from urinary tract infections in the UK. J Med Microbiol. 2019;68(2):161-8.

40. Bader MS, Loeb M, Brooks AA. An update on the management of urinary tract infections in the era of antimicrobial resistance. J Postgrad Med. 2017;129(2):242-58. 